\title{
PCR Techniques for Clonality Assays
}

\author{
Salvador J. Diaz-Cano, M.D., Ph.D., Alfredo Blanes, M.D., Ph.D., and \\ Hubert J. Wolfe, M.D.
}

Clonal overgrowths represent the hallmark of neoplastic proliferations, and their demonstration has been proved useful clinically for the diagnosis of malignant lymphomas based on the detection of specific and dominant immunoglobulin and/or T-cell receptor gene rearrangements. Nonrandom genetic alterations can also be used to test clonal expansions and the clonal evolution of neoplasms, especially analyzing hypervariable deoxyribonucleic acid (DNA) regions from patients heterozygous for a given marker. These tests rely basically on the demonstration of loss of heterozygosity (LOH) resulting from either hemizygosity (nonrandom interstitial DNA deletions) or homozygosity of mutant alleles observed in neoplasms. LOH analyses identify clonal expansions of a tumor cell population, and point to monoclonal proliferation when multiple and consistent $\mathrm{LOH}$ are demonstrated. Based on the methylation-related inactivation of one $\mathrm{X}$ chromosome in female subjects, $\mathrm{X}$-linked markers (e.g., androgen receptor gene) will provide clonality information using LOH analyses after DNA digestion with methylation-sensitive restriction endonucleases. Therefore, both non-X-linked and X-linked analyses give complementary information, related and not related to the malignant transformation pathway respectively. Applied appropriately, these tools can establish the clonal evolution of tumor cell populations (tumor heterogeneity), identify early relapses, distinguish recurrent tumors from other metachronic neoplasms, and differentiate field transformation from metastatic tumor growths in synchronic and histologically identical neoplasms.

Key Words: Clonality $-X$ chromosome inactivationMicrosatellites-Tumor suppressor genes-Tumor progression-Paraffin-embedded tissues.

Diagn Mol Pathol 10(1): 24-33, 2001.

From the Department of Pathology (S.J.D.-C., H.J.W.), Tufts University-New England Medical Center, Boston, Massachusetts, USA; St. Bartholomew's and the Royal London School of Medicine and Dentistry (S.J.D.-C.), London, UK; and the University of Malaga (A.B.), Spain.

Address correspondence and reprint requests to Dr. Salvador J. Diaz-Cano, Department of Histopathology \& Morbid Anatomy, St Bartholomew's and the London NHS Trust, Whitechapel, London E1 1BB, UK (e-mail: s.j.diaz-cano@mds.qmw.ac.uk).
Conceptually, a clone is a group of genetically identical cells descended from one common ancestor. Currently, clonal derivation of cells is the hallmark of neoplasia and strongly implicates acquired somatic mutations giving survival advantage to a clonal cell population (22). Most somatic tissues are polyclonal unless a marked kinetic advantage within a subset of cells causes them to proliferate at the expense of surrounding tissues, resulting in monoclonal tissues.

There are several tests to determine clonality. The markers for clone detection must define a readable and reliable feature linked to a particular cell type and must include karyotypic alterations and single point mutations, among others. The presence of a common and nonrandom alteration in all cells of a tumor confirm clonal origin, but requires in many instances fresh, unfixed tissue-a condition that cannot be met with preneoplasia. Molecular demonstration of a genetic lesion, as small as a point mutation, within all cells is evidence that a common progenitor cell contributed to that mutation. This approach applies only to that fraction of cases in which a "marker" mutation can be identified and fails specifically to identify clonal proliferation that may have taken place before the creation of a specific genetic lesion (Fig. 1). In addition, those particular marker features must also be transmitted to descendant cells and must not induce apoptosis. Under the latter circumstance, any genetic alteration does not result in a dominant clone (15).

A given clone should show proliferative advantages over the remaining nonselected cell population to explain tumor growth. This proliferative advantage does not have to be related to a higher proliferation rate. Apoptotic indices lower than those required normally for regular turnover can increase the total cell number. The most important kinetic feature is the imbalance between cell proliferation and cell loss, ending in cellular overgrowth $(15,20)$. Genetic alterations in a kinetically active tumor cell population lead to progressive clone selection and enhance genetic diversity in a given neoplasm as well. Therefore, the inherent genetic instability within a neoplasm results in heterogeneity in tissues derived from a single cell. As soon as the neoplastic transformation 


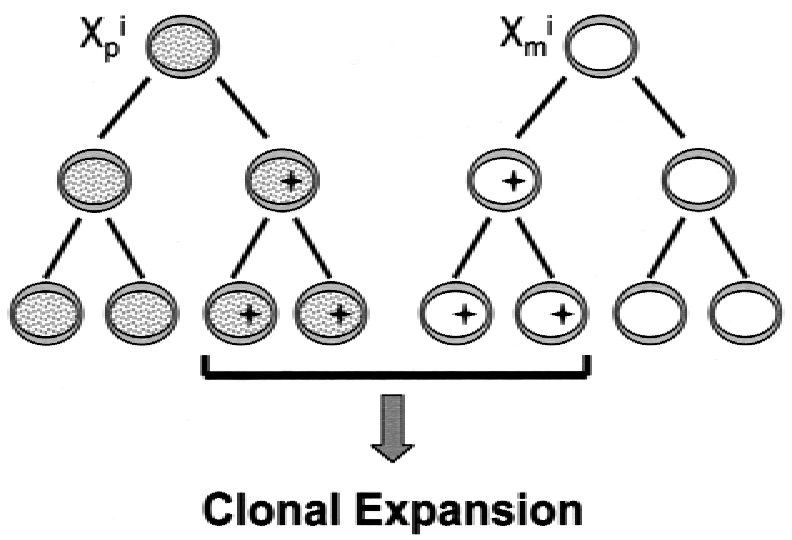

FIG. 1. X-linked and non-X-linked clonality assays provide complementary information. This polyclonal tissue by X-linked analysis would be termed monoclonal for a given marker (black star) after neoplastic-related clonal expansion takes place.

takes place and neoplastic growth ensues, genetically identical cells no longer exist. Nevertheless, certain genetic markers can be used to test clonal expansions within a tumor cell sample. These include gene rearrangement analyses of immunoglobulin genes for B cells and $\mathrm{T}$-cell receptor genes for $\mathrm{T}$ cells $(3,16)$.

We review the principles and requirements of the proper application of polymerase chain reaction (PCR) techniques in the detection of clonality using formalinfixed and paraffin-embedded material. Two main groups of techniques are presented: those based on the analysis of microsatellites (linked or not to $\mathrm{X}$ chromosome) and those related to gene rearrangements. Their potential applications in tumor pathology and pitfalls are summarized briefly. Lastly, some methodological recommendations to obtain reliable results are given.

\section{CLONALITY ASSAYS BASED ON MICROSATELLITE ANALYSIS}

Currently, successful clonality assays using paraffinembedded tissues are based mainly on analyses of widely dispersed, hypervariable regions composed of repetitive deoxyribonucleic acid (DNA) sequences (microsatellites) (36). Several of these microsatellites have been used extensively for DNA fingerprinting and are very useful in genetic linkage analyses, based on their high percentage of heterozygosity in the general population. The loss of heterozygosity ( $\mathrm{LOH}$ ) of a given genetic marker could be linked to loss of tumor suppressor genes (TSG) by DNA deletions (32-34), which would also contribute to the multistep carcinogenesis process selecting cells with growth advantages (44). This progression of genetic events has been found in most inherited cancer syndromes and plays an important role in sporadic cancer development.
Microsatellites have assumed an increasingly important role in this task because of their ubiquity, PCR "typability" (except for [dA] nultimers, whose size polymorphisms are difficult to type by PCR), Mendelian codominant inheritance, and extreme polymorphism $(2,4)$. Microsatellites belong to the family of highly polymorphic and repetitive noncoding DNA sequences. It must be noted that, although widely distributed in the human genome, microsatellites are not uniformly spaced. For example, they are underrepresented in subtelomeric regions of chromosomes. Although their origin and function are not clear (36), their polymorphism has been demonstrated to be very useful in delineating cell lineage $(2,4)$. The different length of these tandemly arranged repeats in the paternally and maternally inherited alleles explains their high polymorphic information content and obvious applications in genome mapping and positional cloning, personal identification, population genetic analysis, and the construction of human evolutionary trees. For clonality purposes, both X-linked and non-X-linked clonality assays can be designed.

Tumor allelotyping $(57,58)$ - the genotypic analysis of all human chromosome pairs for regions of interstitial deletion-is based conceptually on deletions of TSG alleles. The term allelic imbalance is associated closely with $\mathrm{LOH}$ and it is preferred when quantitation of minute DNA amounts is not reliable $(6,7,18,48)$, like in paraffinembedded tissues after microdissection. Both findings are evidence of clonal expansion in a tumor cell population, regardless of the DNA region deleted. Conversely, if the selected polymorphic region is related to known TSG, there is high probability of mutation or function dysregulation of the corresponding gene. Such a situation can explain the growth advantage of the tumor cells carrying that genetic alteration.

\section{X-linked Clonality Analysis}

X-linked clonality analysis can be used only for clonality analysis in informative female subjects, does not assess tumor heterogeneity, and does not provide any data on the precise genetic alteration responsible for clonal proliferation (see Fig. 1). All methods of clonality analysis based on X chromosome inactivation (XCI) include the ability to determine the paternally derived $\mathrm{X}$ chromosome $\left(\mathrm{X}_{\mathrm{p}}\right)$ from the maternally derived one $\left(\mathrm{X}_{\mathrm{m}}\right)$. Many genes on the $\mathrm{X}$ chromosome are polymorphic and permit the distinction between maternally and paternally inherited X chromosomes. Their informativeness is related directly to the frequency of their polymorphism in a population, from $29 \%$ heterozygosity for hypoxanthine phosphoribosyl transferase to more than $90 \%$ for the human androgen receptor gene (26).

According to Lyon's hypothesis (41), all X chromosomes in a cell in excess of one are inactivated on a 
random basis during early embryogenesis. Inactivation of most, but not all, genes on one of the $\mathrm{X}$ chromosomes is mediated by a gene called Xist, and represents a gross imprinting of many genes to achieve a mammalian dose compensation for X-linked genes, which renders maternal and paternal chromosomes nonequivalent functionally. The mechanisms leading to XCI have not been characterized fully, but DNA methylation may maintain the inactive state once it is established. Approximately $60 \%$ of all Cytosine-phosphate-Guanidine $(\mathrm{CpG})$ dinucleotides in the DNA of vertebrates are methylated at the C5 position, but the frequency at particular sites varies between cell types (31). These methylation patterns are transmitted by clonal inheritance through the strong preference of mammalian DNA (cytosine-5) - methyltransferase for hemimethylated DNA. Methylation patterns are established during gametogenesis and early embryogenesis (primordial cell pool of 16-30 cells) (23) and involve, among other DNA regions, promoter regions of alleles on the inactive $\mathrm{X}$ chromosome $\left(\mathrm{X}^{\mathrm{i}}\right)$, whereas alleles on the active $\mathrm{X}$ chromosome $\left(\mathrm{X}^{\mathrm{a}}\right)$ are normally unmethylated (41). Given the small number of embryodestined cells, it reasonable to expect unequal numbers of inactivated $X_{p}$ and $X_{m}$, although $X$ chromosome is inactivated randomly in each cell. Thus the Lyonization ratio $\left(\mathrm{X}_{\mathrm{p}}{ }^{\mathrm{a}} / \mathrm{X}_{\mathrm{m}}{ }^{\mathrm{a}}\right)$ in the population follows a binomial distribution. Allelic variation is determined by the number of progenitor cells at the moment of inactivation (variance observed $=\mathrm{pq} / \mathrm{N}$, where $\mathrm{p}$ and $\mathrm{q}$ are probabilities of inactivating a particular X [both 0.5] and $\mathrm{N}$ is the stem cell pool size). The average Lyonization ratio is close to 50:50 in large cell populations, although individual variation has been found (23), resulting in skewing toward one allele. This is the reason for using as controls for unequal Lyonization the most closely related tissue thought not to be involved in the disease process (14).

The distinction of $\mathrm{X}^{\mathrm{a}}$ from $\mathrm{X}^{\mathrm{I}}$ can be made by gene expression analysis (messenger ribonucleic acid and protein derive only from genes on $\mathrm{X}^{\mathrm{a}}$ ) or can be delineated at the DNA level using methylation-sensitive restriction endonucleases (Fig. 2). The last method can be performed in paraffin-embedded tissues, although it has the absolute requirement of invariable and differential methylation at the polymorphic locus in $\mathrm{X}^{\mathrm{a}}$ and $\mathrm{X}^{\mathrm{i}}$. A perfect $\mathrm{X}$-linked clonality assay would unite highly informative polymorphism with an absolute differential methylation pattern between $\mathrm{X}^{\mathrm{a}}$ and $\mathrm{X}^{\mathrm{i}}$. Therefore, an informative locus amenable to PCR-based detection of nonrandom $\mathrm{XCI}$ must fulfill the following four criteria:

1. High frequency of heterozygosity

2. A site that is methylated differentially in $X^{\mathrm{a}}$ and $X^{\mathrm{i}}$, and thus is subject to differential digestion by methylation-sensitive restriction endonucleases

3. Polymorphic and methylated regions that are in suf-

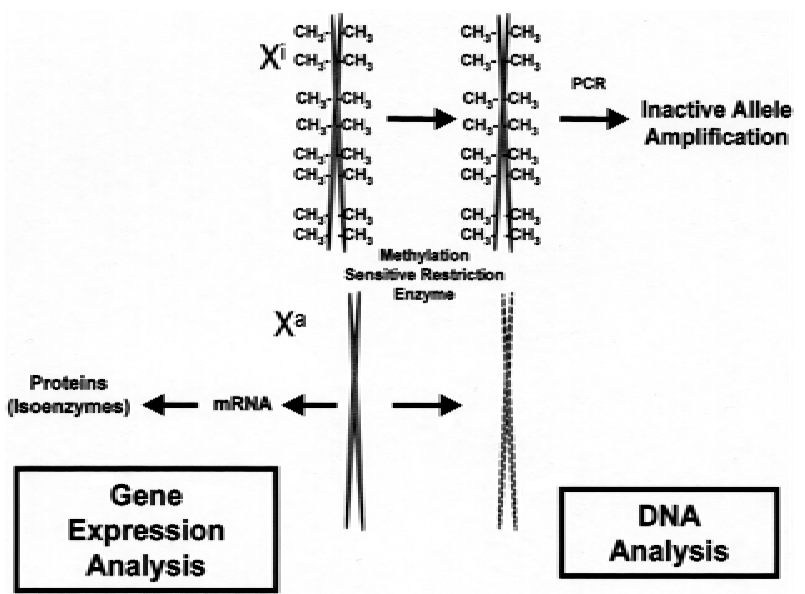

FIG. 2. Dual approach for the analysis of X-chromosome inactivation in females. (Left) Gene expression analyses are based on messenger ribonucleic acid (mRNA) and protein polymorphism from alleles located in the active $X$ chromosome $\left(X^{\mathrm{a}}\right)$. (Right) Conversely, deoxyribonucleic acid (DNA) analysis provides information through the amplification of alleles located on inactive (methylated) $X$ chromosome $\left(X^{i}\right)$ after DNA digestion with methylationsensitive restriction endonucleases. PCR, polymerase chain reaction.

ficient proximity for amplification by one PCR primer set

4. A target that amplifies efficiently and reliably (Fig. 3). Noninvolved normal tissues related closely to the lesion must be tested simultaneously for unequal Lyonization in each particular patient.

Several possible targets have been tested, but currently the best option is the human androgen receptor gene $(55,56)$. This gene has a hypervariable CAG trinucleotide repeat in the coding region of its first exon, located less than 100 base pairs (bp) from four methylation sites recognized by HhaI, and known to be methylated on $X^{i}$ but not on $X^{a}$ (see Fig. 3).

\section{Non-X-linked Clonality Analysis}

These tests rely basically on the demonstration of $\mathrm{LOH}$, resulting from either hemizygosity (nonrandom interstitial DNA deletions) or homozygosity of mutant alleles observed in neoplasms, but only provide information when the genetic marker represents or is linked to a TSG involved in the malignant transformation. These tests represent the first option to study tumor heterogeneity if several samples are taken from a single neoplasm (17), but two main problems must be considered. First, there is no specific sequence of genetic alterations for a given tumor, and therefore several genetic markers must be tested to get information on clonality. Different sequences of genetic alterations have been proposed for several tumor types and locations based on statistical 


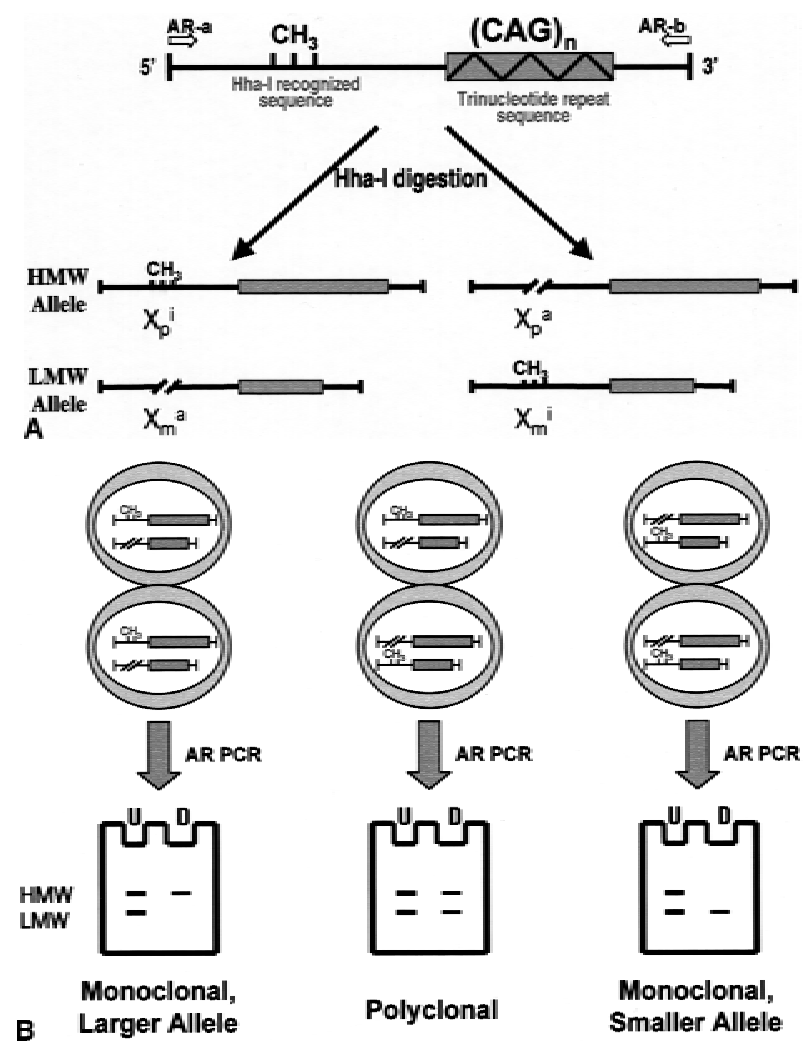

FIG. 3. (A) Structure of the androgen receptor (AR) gene (exon 1). A highly polymorphic CAG repeat sequence location 100 base pairs (bp) downstream of a potentially methylated CG island allows a reliable yield of both components in a single polymerase chain reaction amplification (between 250 and 300 bp). Both alleles can be differentiated easily by size, and can be amplified only from the inactive $X$ chromosome ( $X$, digestion-resistant allele) in the Hhal-digested sample. (B) Allelic patterns of AR gene in monoclonal and polyclonal tissues. The presence of a single allele in Hhal-digested samples (D) in informative cases (two alleles in undigested samples [U]) defines monoclonal tissues (left, right), whereas the retention of both alleles characterizes polyclonal ones (center). Each cell shows the allelic structure after Hhal digestion to emphasize that the polymerase chain reaction amplification comes from the intact allele only (methylated $X$ chromosome). HMW, high molecular weight; LMW, low molecular weight.

analyses of case series $(5,30,57)$, but the frequency of each genetic alteration is variable. Second, the background level of $\mathrm{LOH}$ in normal tissues has been reported to be between $4 \%$ and $20 \%$, regardless of the detection system used $(8,13,51,59)$. A similar LOH frequency must be assumed as background in the evaluation of tumor tissues (51). Considering the worst scenario of all genetic lesions being equally important and frequent (21), the probability of randomly finding coexisting genetic alterations in normal tissues would be $0.2^{2}=4.010^{-2}$ for two genetic loci, $0.2^{3}=8.010^{-3}$ for three genetic loci, and so on. No single genetic alteration of TSG proves by itself that a given proliferation is monoclonal: The $\mathrm{LOH}$ for that particular marker informs only on clonal expansion and cellular selection in genetically heterogeneous tumor cell populations. Only the accumulation of genetic lesions in TSG supports a monoclonal origin of tumors (15), especially if multiple samples from the same tumor show concordant genetic alteration $(14,18)$ (see Methodological Aspects).

\section{CLONALITY ANALYSIS BASED ON SPECIFIC GENE REARRANGEMENTS}

This application will not be covered in detail because it is better known and applicable to malignant lymphomas only. It has been demonstrated to be useful in the diagnosis of malignant lymphomas, in which the presence of homogeneous-appearing lymphoid cell overgrowths are considered histologic evidence of clonal expansion and malignancy $(3,10,16)$.

Committed lymphoid precursors undergo unique sequential assembly of the heavy and light chains of immunoglobulin (B-cell precursors) and T-cell receptor chains (T-cell precursors) during their maturation (16). Their diversity is based on somatic DNA deletions, template-independent nucleotide additions, and specific splicing (39). These processes involve, in the case of immunoglobulin heavy chain, three highly variable regions, complementarity-determining regions, which separate four framework regions (FR) (10). The locations of DNA breaks are determined by short DNA sequences (7 and $9 \mathrm{bp}$ ) recognized by the recombination-activating proteins. The presence of length-specific spacer sequences provides the right splicing and determines the DNA rearrangement order (e.g., D-J and V-DJ in the case of immunoglobulin). Template-independent nucleotide additions give the final DNA rearrangement of immunoglobulin or T-cell receptor chains. All gene rearrangements in lymphoid precursors take place only in cells expressing terminal deoxynucleotidyl transferase (16).

The PCR design for gene rearrangement detection has to consider the special situation of DNA sequence addition and deletion. It is particularly important for the primer binding regions, which must be located in less variable sequences (FR regions for immunoglobulin gene rearrangement) to avoid failed amplification resulting from their loss. This factor also helps to explain false-negative results in the detection of clonal rearrangement in well-differentiated lymphoid neoplasms (related to gene hypermutability). Complete rearrangements may be associated with the loss of inner FR regions (in B cells) and with $\delta$-chain (in T cells), the most sensitive primer binding regions to detect early rearrangement. Therefore, a broad approach is recommended for detecting clonal rearrangement, including at least two different set of primers (FR III-FR IV and FR I-FR IV) in B-cell lymphoid lesions (16), and several primer sets 
for the T-cell receptor $\gamma$-chain in T-cell lymphoid lesions (3).

All PCR-based analyses must be run with appropriate controls, including internal positive (provided by nonspecific amplifications of locus-homologous sequences), positive (lymphoid proliferation with clonal rearrangement), negative (polyclonal lymphoid proliferation), and technique efficiency (mixture of monoclonally and polyclonally rearranged DNA tested previously) (16). The last control is needed essentially for the clinical application of these tests when sensitivity is an absolute requirement (16). Their interpretation must also consider the presence of false-positive and false-negative cases, and their causes $(3,16)$. It should be emphasized that clonal gene rearrangement does not mean malignancy, because even some benign conditions can show it. Furthermore, well-defined monoclonal PCR bands can be observed when the lymphoid DNA template is present in trace amounts. Nonamplifiable DNA may result from the failure of PCR amplification for technical reasons (contamination, highly fragmented DNA, etc.) or when the presence of rearranged DNA is less than the sensitivity level (approximately $1 \%$ of the total cell population). Lastly, oligoclonal proliferation (especially in immunodeficiency-related lymphomas) can result in smear patterns from overlapped clonal bands.

\section{GENERAL INTERPRETATION CRITERIA AND QUALITY CONTROLS OF CLONALITY MICROSATELLITE ANALYSES}

Two complementary aspects are evaluated with Xlinked and non-X-linked clonality analyses (see Fig. 1). XCI takes place early during embryologic development, usually before and unrelated to any genetic event involved in the malignant transformation. Therefore, it is really informing about clonality in tumors and precancerous conditions, although it is not able to inform on the specific molecular alterations. Different polymorphic regions have been related to TSG (recessive trait genes). Therefore, LOH analyses enable us to study the molecular pathways involved in the malignant transformation, and to test clonal expansion and tumor heterogeneity if various molecular markers are assessed in samples from different tumor areas. Moreover, the correlation of these molecular markers with other pathologic parameters (like tumor cell invasion, nuclear grade, proliferation indices, etc.) can help us to identify high-risk patients and to understand the process of multistep carcinogenesis. All nonrandom gene rearrangements, as markers of malignancy, fulfill the criteria mentioned for $\mathrm{LOH}$ analyses.

Any molecular analysis must be run with appropriate controls, including known positive (monoclonal proliferations, homozygous for the marker) and negative (polyclonal proliferations, heterozygous for the marker) controls, and from embryologically related tissues for $\mathrm{XCI}$ analysis to exclude a skewed Lyonization ratio (18, 20,43). The PCR approach for microsatellite analysis (including both clonality assays) must amplify the right locus and accurately identify informative patients (two different alleles present in control tissues; Fig. 4). This issue becomes especially important for microsatellite analysis when the presence of extra bands is not exceptional, especially in cases of internal labeling. A sine qua non requirement for clonality analysis is the identification of a polymorphic locus in the normal control (see Fig. 4). In every case, the tumor sample must be compared with normal controls from the same patient to test patient heterozygosity for the marker-a feature that must be maintained in HhaI-digested samples in tests based on DNA methylation, such as XCI assays (Fig. 5). Patients with two identical alleles or showing skewed Lyonization in control tissues should be considered noninformative and should be excluded from clonality evaluation. Another cause of noninformative cases is the anomalous expansion or reduction of tandem repeats resulting from microsatellite instability that results in extra bands (see Fig. 4). True new bands, as true evidence of microsatellite instability, are located normally in the expected size range (usually approximately $100 \mathrm{bp}$ ), above or below the expected PCR product.

Therefore, $\mathrm{LOH}$ and allelic imbalances of gene loci can be interpreted as evidence of monoclonal proliferation (for X-linked assays of templates digested by methylation-sensitive restriction enzyme, such as HhaI) or clonal expansion (for non-X-linked assays, including gene rearrangements), if noninformative cases have been excluded previously (see Fig. 1 and 4). Positive allelic

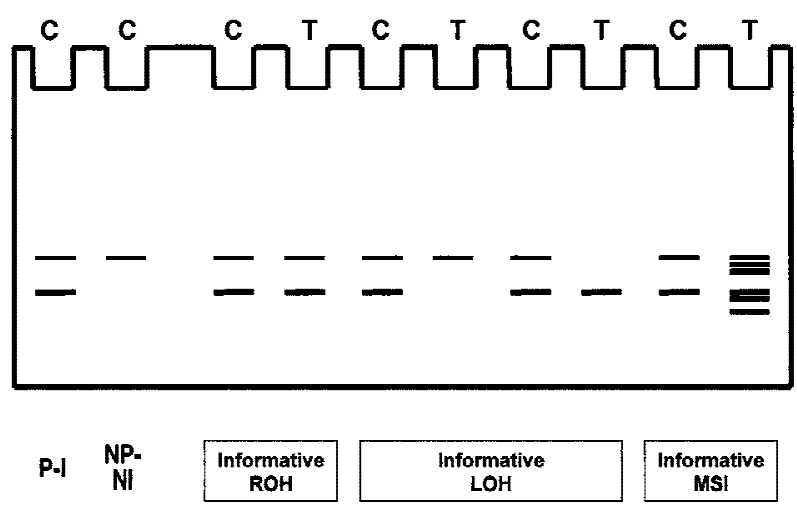

FIG. 4. Polymerase chain reaction-based analysis of microsatellites in clonality assays. Allelic polymorphism (P) in any given control identifies informative (I) patients (left). The tumor $(\mathrm{T})$-control $(\mathrm{C})$ comparison allows case classification as normal (retention of heterozygosity $[\mathrm{ROH}]$ ) or abnormal (loss of heterozygosity [ $\mathrm{LOH}]$ and microsatellite instability [MSI]). Cases showing MSI could be either monoclonal or polyclonal and should be excluded from clonality assays. NP, no polymorphism; NI, noninformative patient. 


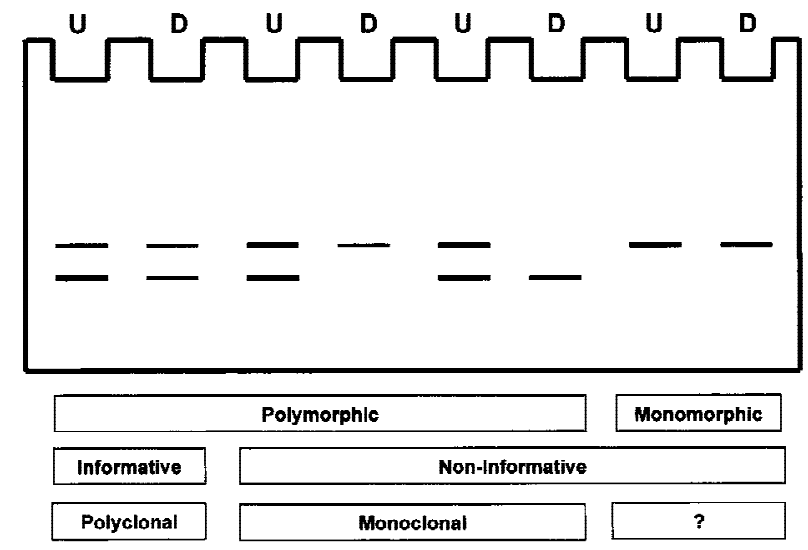

FIG. 5. Gel patterns of androgen receptor (AR) alleles from controls. Only polyclonal controls (two-band pattern on Hhal-digested samples [D]) from tissues with ARallelic polymorphism (two-band pattern on Hhalundigested samples [U]) are informative for X-linked clonality assays. Monoclonal controls (even from polymorphic tissues) and tissues with AR-allelic monomorphism lack informativeness for clonality purposes.

imbalances are determined case by case, in relation to the densitometric allelic ratio in the normal control (43), which requires a threshold of at least 4:1 in skewed data. The host cell contamination of tumor samples could give false heterozygous results that would require careful microdissection and microscopic control of the sample collection.

\section{APPLICATIONS AND PITFALLS}

Clonality assays can be useful in the analysis of different biologic processes. They have been used mainly in the study of malignant transformation and tumor progression. At this level, the complementary information provided by $\mathrm{X}$-linked and non-X-linked markers contributes to the definition of the real nature of the lesion, as mentioned earlier. Monoclonal patterns would support the neoplastic nature, although they have been described in other proliferative processes such as aggressive fibromatosis (1) or focal nodular hyperplasia of the liver (25). Similarly, the polyclonal patterns reported in sacrococcygeal cystic teratomas suggest their hamartomatous nature, opposed to the monoclonal immature teratoma (54). The acquisition of additional genetic deletions in certain histologic areas favors a molecular progression as reported for the adenoma-carcinoma sequence in colon (52), or sporadic neuroendocrine tumors of the pancreas (45). The acquisition of genetic changes has been considered evidence of molecular progression that also results in tumor heterogeneity. Tests demonstrating those abnormalities only prove clonal expansions, but would support monoclonality if several markers from different tumor areas show concordant genetic alterations $(15,18)$.
The identity of synchronic or metachronic tumors can be tested at the molecular level using these clonality markers. Both extremes have been reported in tumors. Some coexistent tumors have revealed the same pattern of genetic markers (both X linked and non-X linked), suggesting that a common progenitor contributed to those lesions, and thus supporting a multifocal rather than a multicentric origin. This particular situation has been demonstrated in bladder tumors $(18,53)$, human immunodeficiency virus-associated Kaposi's sarcoma (47), disseminated peritoneal leiomyomatosis (46), or multifocal C-cell hyperplasias and nodular adrenal medullary hyperplasias associated with multiple endocrine neoplasia $2 \mathrm{~A}$ (20a). In the case of malignant neoplasms, this shared genetic alteration would support a metastatic origin for the tumors (11), or a common cellular origin for biphasic neoplasms (60). Opposite findings have been reported for prostatic tumor foci when the heterogeneous genetic composition suggests either an independent evolution of those foci from a common progenitor or a completely different origin $(28,29)$.

Lastly, any well-characterized genetic alteration can be used for the early detection of recurrences, both local and systemic. These alterations pick clonal expansion from selected groups of cells, and they have been proposed as tools to study the resection margins in conservative surgery, as reported for head and neck squamous cell carcinomas (35). Likewise, the presence of minimal residual disease can be defined better at the molecular level by detecting circulating tumor cells with specific gene alterations, more frequently gene rearrangements, as reported for malignant lymphoma (16) or sarcomas of the Ewing family (12).

These molecular studies should be interpreted with caution. Some considerations should be made to set the proper value of these techniques. These considerations include tumor cell heterogeneity, sample size, tissue control, restriction enzyme digestion and abnormal methylation, and artifactual allelic dropout.

\section{Tumor Cell Heterogeneity}

Tumor cell heterogeneity is linked to genetic instability and biologic progression. This genetic heterogeneity is often reflected in phenotypic expression. Examples include the presence of a $\mathrm{Ki}$-ras point mutation in carcinomatous areas from adenomatous polyposis coli (APC)mutated sporadic colorectal adenomas (52). Similarly, mutations in cell cycle regulators (e.g., tumor protein 53, retinoblastoma, cyclins, cyclin-dependent kinases, and cyclin-dependent kinase inhibitors) have been related to either proliferative advantages or apoptotic dysregulation (9). So, it can explain their association with tumor grade as far as nuclear atypia (pleomorphism, chromatin features, and size variability) is expression of both proliferation and apoptosis. Tumor heterogeneity must be 
studied using several tumor samples of appropriate size from each tumor (see the following section).

\section{Sample Size}

Sample size is a limiting factor. To increase sample homogeneity and to avoid normal cell contamination, very small samples (even single cells) have been used in genetic analyses. However, the lower the number of cells, the higher the probability of false monoclonal patterns based on inadequate sampling. Although early XCI takes place at random and usually gives a chessboard pattern, small cell populations descended from a common stem cell may grow together like a clone (patch size concept or contiguous cellular regions of the same lineage). For this reason, monoclonal XCI patterns are reported in breast lobules when studied from single samples. Multiple samplings from different areas and sample sizes larger than 100 cells or $0.25 \mathrm{~mm}^{2}$ can avoid this problem.

The sample size in tumor cell analysis is, therefore, an important parameter, particularly in light of tumor cell heterogeneity. Microdissection techniques allow us to pick up very small samples selectively, which can show false cellular homogeneity, based on LOH or allelic imbalance. If the tumor cell populations selected for molecular analysis are taken before they become a biologically prominent component (with proliferative or invasive advantages), the results obtained may be confusing and irrelevant clinically, and need to be evaluated in the proper biologic context. This would be the case with microheterogeneity in tumors that tend to give disparate results with meanings that remain essentially unknown. Except for intraepithelial proliferation, all cell samples with microdissection provide target cell-rich samples with a varying degree of host cell contamination (including stromal, inflammatory, and endothelial cells). Taking all these factors into consideration, multiple samples from the same case should always be studied, and assays should be performed in duplicate before accepting the results as relevant.

\section{Tissue Control}

Tissue control from the same patient is an absolute requirement to test patient heterozygosity for a particular TSG marker (as mentioned earlier; see Fig. 5 and Fig. 6). Ideally, embryologically related tissues are the best to show a reliable X-linked clonality pattern. Oncogene genetic changes such as RAS point mutations that are not found in the germline are excluded from this approach and do not require heterozygosity control.

\section{Restriction Enzyme Digestion and Abnormal Methylation}

Every single step for all molecular tests must be controlled for completion to avoid false results. Appropriate

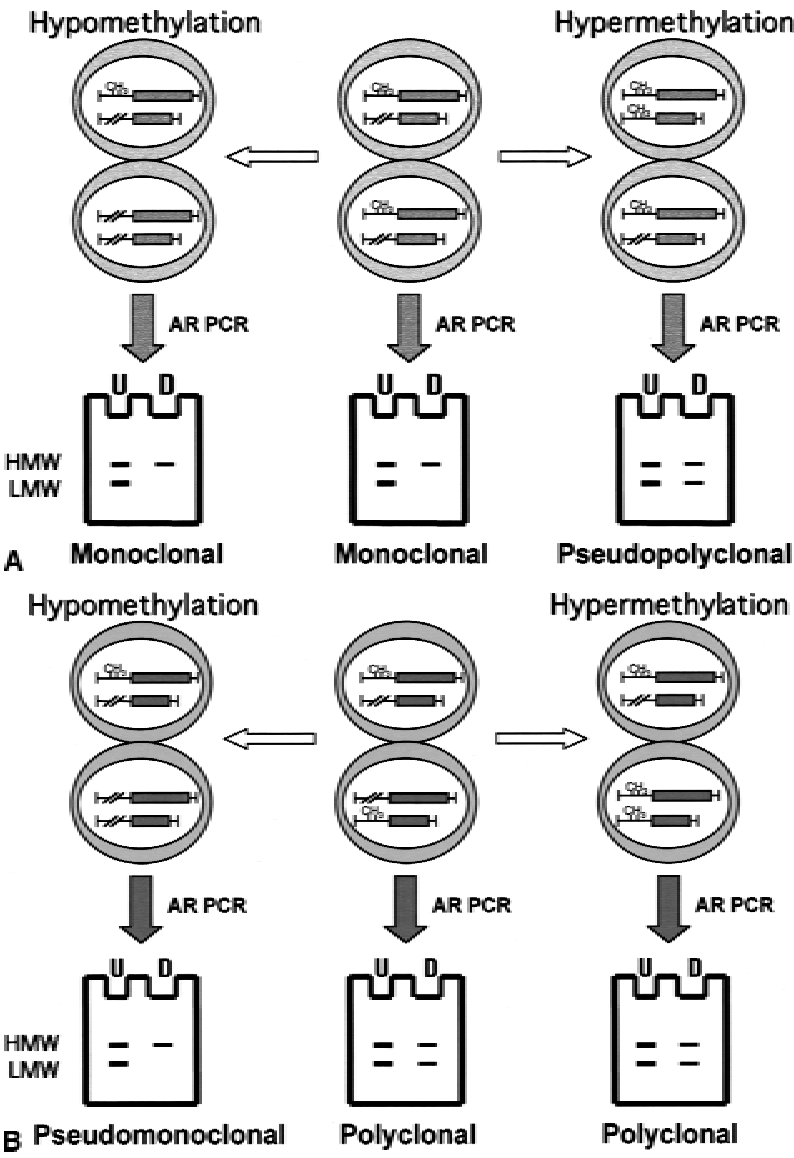

FIG. 6. Methylation level and allelic patterns of androgen receptor (AR). (A) Monoclonal tissues would only change the clonality pattern if the amplified AR locus was hypermethylated. A pseudopolyclonal pattern would be revealed on gel electrophoresis (right). (B) Polyclonal tissues would only change the clonality pattern if the amplified AR locus was hypomethylated. A pseudomonoclonal pattern would be revealed on gel electrophoresis (B). PCR, polymerase chain reaction; U, Hhal-undigested sample; D, Hhal-digested sample; HMW, high molecular weight; LMW, low molecular weight.

controls must be run, especially for restriction enzyme digestions. Because XCI analysis is based on differential DNA methylation of one allele from X-chromosome genes (e.g., human androgen receptor gene) and relies on endonuclease digestion by methylation-sensitive restriction enzymes, suboptimal enzymatic digestion provides a changed clonality pattern in the case of monoclonal tissues. Likewise, abnormal methylation provides potentially the same false result in the case of hypermethylation, whereas hypomethylation could affect the clonality pattern in polyclonal tissues giving a pseudomonoclonal one (see Fig. 6). Additionally, methylation abnormalities occur during the course of malignant transformation (37). Hypomethylation has been described in relation to increased proliferation during early stages of neoplasms, whereas hypermethylation has been linked to 
late stages associated with a higher mutation rate and tumor progression (42).

\section{Artifactual Allelic Dropout}

PCR bias against one allele (especially the larger one) can result in preferential amplification of the other allele (usually the smaller) $(24,49)$. An appropriate extraction method providing DNA of enough quality $(3,19)$, and PCR designs including both long denaturation and extension during the first three cycles, and 7-deaza-deoxyguanidine triphosphate (dGTP) in the amplification mixture to improve the amplification of CG-rich DNA regions reasonably avoids that bias $(18,20,49)$.

\section{METHODOLOGIC ASPECTS}

Formalin-fixed, paraffin-embedded tissues are analyzed more easily at the DNA level, resulting in part from the better preservation of this nucleic acid. Therefore, any PCR-based technique applied to this material should consider DNA extraction, DNA modification (such as restriction enzyme digestion), target amplification, and adequate gel resolution of the products.

\section{DNA Extraction Process}

Several chemical modifications are induced in tissues by fixation and processing, including cross-linking between basic amino acids of proteins and the amino groups on DNA bases. Nonspecific amplifications are caused mainly by primer-independent, but DNA polymerase- and cycling-dependent, incorporation of nucleotides into DNA, possibly related to DNA repair and/or internal priming (40). This is the reason for complete and intense protein digestion before DNA purification (19). Denaturing reagents can break the cross-linked strands but in turn provide short-length DNA strands, precluding their use in protocols that require DNA of 250 to $500 \mathrm{bp}$ in length. The general DNA quality of the extracted DNA should be tested by gel electrophoresis of the protein-digested sample, universal DNA amplification using degenerated oligonucleotide primer-PCR, or amplifying $\beta$-globin gene with primer sets at least $100 \mathrm{bp}$ lengthier than the final DNA target.

The easiest protocol giving the highest DNA quality is 55 to $60^{\circ} \mathrm{C}$ prolonged proteinase $\mathrm{K}$ digestion (5-7 days, with every-day enzyme replacement) (19). The standard phenol-chloroform purification protocol results in the best contaminant-free DNA for any PCR application. Negative amplification resulting from sample contamination (specific amplification lacking with no primer dimers) can be avoided by diluting the sample.

\section{DNA Modifications}

Certain applications need original DNA strands, such as those based on the genomic imprinting of XCI. The presence of methylated cytosine can be tested by sample digestion by methylation-sensitive restriction endonucleases. In any case, appropriate internal control should be included to prove complete digestion. The samples already show smear patterns, and even they may be undetectable using gel electrophoresis (especially for microdissected samples). A logical way to accomplish this issue is to include DNA mimickers in every sample undergoing restriction enzyme digestion. These mimickers are normally viral DNA (such as phages) and should fulfill some requirements. They must be linear and double stranded (like human genomic DNA), and they must contain base sequences recognized by the tested enzyme with reliable pre- and postdigestion patterns. In addition, no sequence similarity able to give nonspecific amplification in further PCR should be present. Xho-Ilinearized $\phi X 174 \mathrm{RII}$ phage represents an ideal mimic for HhaI digestion used for XCI analysis.

$\mathrm{XCI}$ analysis tests the differential methylation level in a $\mathrm{CpG}$ island approximately $100 \mathrm{bp}$ upstream of the $\mathrm{CAG}$ repeat (56). Different methylation-sensitive restriction enzymes have been used, especially HhaI and HpaII. The first provides more reliable results because of its activity with single-strand DNA (that activity has not been demonstrated for HpaII). We must keep in mind that the embedding process partly denatures DNA and, therefore, single-strand DNA is a normal component in archival material.

\section{Target Amplification by PCR and Gel Resolution}

All PCR methods should consider the appropriate conditions regarding $\mathrm{Mg}^{2+}$ and primer concentrations, nucleotide concentration, number of cycles in case of PCR-based quantitative analyses, PCR product labeling, and detection methods. The standards for all PCR technique need specific optimization for each set of primer according to $\mathrm{Mg}^{2+}$ (normally $1.5 \mathrm{mM}$ ) and nucleotide concentration (in the $50-\mu \mathrm{M}$ range for microsatellite analyses). PCR cycling conditions should always consider the number of cycles to avoid product saturation unacceptable for any quantitative PCR design; in general, between 25 and 30 cycles give adequate amplification in accordance with the initial DNA concentration. We optimized experimentally the conditions for PCR as follows: the reactions were run with $1.5 \mathrm{mM} \mathrm{MgCl}_{2}$, using $0.3 \mu \mathrm{M}$ each primer, $200 \mu \mathrm{M}$ each dNTP (including 7-deaza-dGTP instead of dGTP) and $1 \mu \mathrm{L}$ template. A long denaturation (4 minutes) was used in the first three cycles, the annealing temperature was $55^{\circ} \mathrm{C}$, and the number of cycles was optimized experimentally to 28. A "hot start" (addition of primers to mixtures kept at $85^{\circ} \mathrm{C}$ ) should also be included to facilitate the complete denaturation of DNA strands with high CG content in the initial amplifications $(18,20,43)$. 
DNA samples from microdissected tissues are not ideal for reliable quantification and are generally run with unknown target DNA concentration. So, relative quantification of both allelic bands must be taken into consideration to determine whether LOH (or allelic imbalance) is present. This issue brings us to consider the labeling and detection method. ${ }^{32} \mathrm{P}$ - and ${ }^{33} \mathrm{P}$-based radiolabeling represent the standard protocols, including both external (one primer is $5^{\prime}$ labeled) and internal (labeled nucleotide in the PCR mixture) methods. Although the latter usually gives more background, it permits the highest sensitivity for microdissected paraffin-embedded samples. Allelic separation can be achieved by running the samples far enough into high-resolution denaturing polyacrylamide gels (variable concentrations of formamide and urea). Some other detection methods have been used, including fluorescent labeling $(6,7)$ and silver staining of PCR products (38). The highest sensitivity is achieved by radioisotopic methods, which remains the standard for molecular detection of genetic alterations, especially in formalin-fixed, paraffin-embedded material. Additionally, the ratio between signal and initial DNA amount is highly variable for silver-stained gels, making the applications of this technique less reliable for quantification. Different technical approaches have been used to detect interstitial DNA deletion and single base changes (mutations/polymorphisms), including singlestrand conformational polymorphism, denaturant gradient gel electrophoresis, mutant allele-specific amplification, ribonuclease (RNase) protection, etc. $(27,50)$. Although the final proof for any mutation must be direct sequencing, one of the most sensitive methods for detecting single base changes is PCR/denaturant gradient gel electrophoresis, which is able to distinguish DNA strands differing in only one base $(3,18)$.

Lastly, the linear ratio between radioactive emission and signal deposition can be maintained by film preflashing to get a 0.1 to $0.2-\mathrm{OD}$ unit absorbance increase at 540 $\mathrm{nm}$ in the preflashed film. In addition, signal stabilization during autoradiogram development requires $-70^{\circ} \mathrm{C}$ storage. The allelic ratio has to be quantitated in normalized samples to exclude any potential contamination with normal tissue (43). At that level, different computer software is available to aid in analysis.

Acknowledgment: Presented in part at the Xth International Congress of Histochemistry and Cytochemistry, Kyoto, Japan, August 18-23, 1996; and at the XXIst International Congress of the International Academy of Pathology, Budapest, Hungary, October 20-25, 1996.

\section{REFERENCES}

1. Alman BA, Pajerski ME, Diaz-Cano S, Corboy K, Wolfe HJ. Aggressive fibromatosis (desmoid tumor) is a monoclonal disorder. Diagn Mol Pathol 1997;6:98-101.
2. Boland CR. Setting microsatellites free. Nat Med 1996;2:972-4.

3. Brady SP, Magro CM, Diaz-Cano SJ, Wolfe HJ. Analysis of clonality of atypical cutaneous lymphoid infiltrates associated with drug therapy by PCR/DGGE. Hum Pathol 1999;30:130-6.

4. Brentnall TA. Microsatellite instability. Shifting concepts in tumorigenesis. Am J Pathol 1995;147:561-3.

5. Califano J, van der Riet $\mathrm{P}$, Westra W, et al. Genetic progression model for head and neck cancer: implications for field cancerization. Cancer Res 1996;56:2488-92.

6. Cawkwell L, Bell SM, Lewis FA, Dixon MF, Taylor GR, Quirke P. Rapid detection of allele loss in colorectal tumours using microsatellites and fluorescent DNA technology. Br J Cancer 1993; 67:1262-7.

7. Cawkwell L, Li D, Lewis FA, Martin I, Dixon MF, Quirke P. Microsatellite instability in colorectal cancer: improved assessment using fluorescent polymerase chain reaction. Gastroenterology 1995;109:465-71.

8. Chen LC, Kurisu W, Ljung BM, Goldman ES, Moore DI, Smith HS. Heterogeneity for allelic loss in human breast cancer. $J$ Natl Cancer Inst 1992;84:506-10.

9. Cordon-Cardo C. Mutations of cell cycle regulators. Biological and clinical implications for human neoplasia. Am J Pathol 1995; 147:545-60.

10. Cossman J, Zehnbauer B, Garrett CT, et al. Gene rearrangements in the diagnosis of lymphoma/leukemia. Guidelines for use based on a multiinstitutional study. Am J Clin Pathol 1991;95:347-54.

11. Cuatrecasas M, Matias-Guiu X, Prat J. Synchronous mucinous tumors of the appendix and the ovary associated with pseudomyxoma peritonei. A clinicopathologic study of six cases with comparative analysis of c-Ki-ras mutations. Am J Surg Pathol 1996;20:739-46.

12. de Alava E, Lozano MD, Patino A, Sierrasesumaga L, Pardo-Mindan FJ. Ewing family tumors: potential prognostic value of reverse-transcriptase polymerase chain reaction detection of minimal residual disease in peripheral blood samples. Diagn Mol Pathol 1998;7:152-7.

13. Deng G, Lu Y, Zlotnikov G, Thor AD, Smith HS. Loss of heterozygosity in normal tissue adjacent to breast carcinomas. Science 1996;274:2057-9.

14. Diaz-Cano SJ. Designing a molecular analysis of clonality in tumours. J Pathol 2000;191:343-4.

15. Diaz-Cano SJ. Clonality studies in the analysis of adrenal medullary proliferations: application principles and limitations. Endocr Pathol 1998;9:301-16.

16. Diaz-Cano S. PCR-based alternative for diagnosis of immunoglobulin heavy chain gene rearrangement: principles, practice, and polemics. Diagn Mol Pathol 1996;5:3-9.

17. Diaz-Cano SJ, Blanes A. Influence of intratumor heterogeneity in the interpretation of marker results in pheochromocytomas. $J$ Pathol 1999;189:627-8.

18. Diaz-Cano SJ, Blanes A, Rubio J, Matilla A, Wolfe HJ. Molecular evolution and intratumor heterogeneity by topographic compartments in muscle-invasive transitional cell carcinoma of the urinary bladder. Lab Invest 2000;80:279-89.

19. Diaz-Cano SJ, Brady SP. DNA extraction from formalin-fixed, paraffin-embedded tissues: protein digestion as a limiting step for retrieval of high-quality DNA. Diagn Mol Pathol 1997;6:342-6.

20. Diaz-Cano SJ, de Miguel M, Blanes A, Tashjian R, Galera H, Wolfe HJ. Clonality as expression of distinctive cell kinetics patterns in nodular hyperplasias and adenomas of the adrenal cortex. Am J Pathol 2000;156:311-9.

20a. Diaz-Cano SJ, de Miguel M, Blanes A, Tashjian R, Galera H, Wolfe HJ. Clonal patterns in phaechromocytomas and MEN-2A adrenal medullary hyperplasias: histologic and kinetic correlates. J Pathol 2000;192:221-8.

21. Diaz-Cano SJ, Wolfe HJ. Clonality in Kaposi's sarcoma. $N$ Engl J Med 1997;337:571-2.

22. Fialkow PJ. Clonal origin of human tumors. Biochim Biophys Acta 1976;458:283-321.

23. Fialkow PJ. Primordial cell pool size and lineage relationships of five human cell types. Ann Hum Genet 1973;37:39-48.

24. Findlay I, Matthews P, Quirke P. Multiple genetic diagnoses from 
single cells using multiplex PCR: reliability and allele dropout. Prenat Diagn 1998;18:1413-21.

25. Gaffey MJ, Iezzoni JC, Weiss LM. Clonal analysis of focal nodular hyperplasia of the liver. Am J Pathol 1996;148:1089-96.

26. Gilliland DG, Blanchard KL, Levy J, Perrin S, Bunn HF. Clonality in myeloproliferative disorders: analysis by means of the polymerase chain reaction. Proc Natl Acad Sci U S A 1991;88:6848-52.

27. Housman DE. DNA on trial- the molecular basis of DNA fingerprinting. $N$ Engl J Med 1995;332:534-5.

28. Hugel A, Wernert N. Loss of heterozygosity (LOH), malignancy grade and clonality in microdissected prostate cancer. Br J Cancer 1999;79:551-7.

29. Jenkins RB, Qian J, Lieber MM, Bostwick DG. Detection of c-myc oncogene amplification and chromosomal anomalies in metastatic prostatic carcinoma by fluorescence in situ hybridization. Cancer Res 1997;57:524-31.

30. Jones PA, Droller MJ. Pathways of development and progression in bladder cancer: new correlations between clinical observations and molecular mechanisms. Semin Urol 1993;11:177-92.

31. Kappler JW. The 5-methylcytosine content of DNA: tissue specificity. J Cell Physiol 1971;78:33-6.

32. Knudson AG. Antioncogenes and human cancer. Proc Natl Acad Sci U S A 1993;90:10914-21.

33. Knudsen AG Jr. Mutation and cancer: statistical study of retinoblastoma. Proc Natl Acad Sci U S A 1971;68:820-3.

34. Knudsen AG Jr, Hethcote HW, Brown BW. Mutation and childhood cancer: a probabilistic model for the incidence of retinoblastoma. Proc Natl Acad Sci U S A 1975;72:5116-20.

35. Koch WM, Brennan JA, Zahurak M, et al. p53 Mutation and locoregional treatment failure in head and neck squamous cell carcinoma. J Natl Cancer Inst 1996;88:1580-6.

36. Koreth J, O'Leary JJ, McGee JOD. Microsatellites and PCR genomic analysis. J Pathol 1996;178:239-48.

37. Laird PW, Jaenisch R. DNA methylation and cancer. Hum Mol Genet 1994;3:1487-95.

38. Lau DH, Yang B, Hu R, Benfield JR. Clonal origin of multiple lung cancers: K-ras and p53 mutations determined by nonradioisotopic single-strand conformation polymorphism analysis. Diagn Mol Pathol 1997;6:179-84.

39. Lewis SM, Wu GE. The origin of V(D)J recombination. Cell 1997; 88:159-62.

40. Long AA, Komminoth P, Lee E, Wolfe HJ. Comparison of indirect and direct in-situ polymerase chain reaction in cell preparations and tissue sections. Detection of viral DNA, gene rearrangements and chromosomal translocations. Histochemistry 1993;99:151-62.

41. Lyon MF. Some milestones in the history of X-chromosome inactivation. Annu Rev Genet 1992;26:16-28.

42. Magewu AN, Jones PA. Ubiquitous and tenacious methylation of the $\mathrm{CpG}$ site in codon 248 of the 553 gene may explain its frequent appearance as a mutational hot spot in human cancer. Mol Cell Biol 1994; $14: 4225-32$.

43. Mutter GL, Boynton KA. X chromosome inactivation in the nor- mal female genital tract: implications for identification of neoplasia. Cancer Res 1995;55:5080-4.

44. Nowell PC. The clonal evolution of tumor cell populations. Science 1976;194:23-8.

45. Perren A, Roth J, Muletta-Feurer S, et al. Clonal analysis of sporadic pancreatic endocrine tumours. J Pathol 1998;186:363-71.

46. Quade BJ, McLachlin CM, Soto-Wright V, Zuckerman J, Mutter GL, Morton CC. Disseminated peritoneal leiomyomatosis. Clonality analysis by $\mathrm{X}$ chromosome inactivation and cytogenetics of a clinically benign smooth muscle proliferation. Am J Pathol 1997; 150:2153-66.

47. Rabkin CS, Janz S, Lash A, et al. Monoclonal origin of multicentric Kaposi's sarcoma lesions. N Engl J Med 1997;336:988-93.

48. Randerson J, Cawkwell L, Jack A, et al. Fluorescent polymerase chain reaction of a panel of CA repeats on chromosome 6 in the indolent phase of follicular centre cell lymphoma. $\mathrm{Br} J$ Cancer 1996;74:942-6.

49. Ray PF, Handyside AH. Increasing the denaturation temperature during the first cycles of amplification reduces allele dropout from single cells for preimplantation genetic diagnosis. Mol Hum Reprod 1996;2:213-18.

50. Rosenthal N. Molecular medicine. Recognizing DNA. $N$ Engl $J$ Med 1995;333:925-7.

51. Sager R. Tumor suppressor genes: the puzzle and the promise. Science 1989;246:1406-12.

52. Shibata D, Schaeffer J, Li ZH, Capella G, Perucho M. Genetic heterogeneity of the $\mathrm{c}-\mathrm{K}$-ras locus in colorectal adenomas but not in adenocarcinomas. J Natl Cancer Inst 1993;85:1058-63.

53. Sidransky D, Frost P, Von Eschenbach A, Oyasu R, Preisinger AC, Vogelstein B. Clonal origin bladder cancer. N Engl J Med 1992; 326:737-40.

54. Sinnock KL, Perez-Atayde AR, Boynton KA, Mutter GL. Clonal analysis of sacrococcygeal "teratomas." Pediatr Pathol Lab Med 1996;16:865-75.

55. Sleddens HF, Oostra BA, Brinkmann AO, Trapman J. Trinucleotide (GGN) repeat polymorphism in the human androgen receptor (AR) gene. Hum Mol Genet 1993;2:493.

56. Sleddens HF, Oostra BA, Brinkmann AO, Trapman J. Trinucleotide repeat polymorphism in the androgen receptor gene (AR). Nucl Acid Res 1992;20:1427.

57. Vogelstein B, Fearon ER, Hamilton SR, et al. Genetic alterations during colorectal-tumor development. N Engl J Med 1988;319: $525-32$.

58. Vogelstein B, Fearon ER, Kern SE, et al. Allelotype of colorectal carcinomas. Science 1989;244:207-11.

59. Wolman SR, Heppner GH. Genetic heterogeneity in breast cancer. J Natl Cancer Inst 1992;84:469-70.

60. Zhuang Z, Lininger RA, Man YG, Albuquerque A, Merino MJ, Tavassoli FA. Identical clonality of both components of mammary carcinosarcoma with differential loss of heterozygosity. Mod Pathol 1997;10:354-62. 